\title{
Procesamiento traumatogénico del abuso sexual infantil en niñas y su relación con variables victimológicas
}

\section{Traumatogenic processing of child sexual abuse and its relation to victimological variables}

\author{
Hugo Plaza Villarroel ${ }^{1}$ \\ Escuela de Psicología, Universidad Santo Tomás, Viña del Mar, Chile \\ Christian Beraud Fernández \\ Centro Trafún, ONG Paicabí, Viña del Mar, Chile \\ Catalina Valenzuela Arancibia \\ ONG CECAS, Viña del Mar, Chile
}

(Rec: septiembre 2014 - Acep: noviembre 2014)

\begin{abstract}
Resumen
Se indaga la relación entre el procesamiento traumatogénico de experiencias de violencia sexual en niñas, con características de la victimización sufrida. Una muestra de 40 niñas víctimas de violencia sexual respondió una escala de sentimientos de estigmatización, indefensión, traición y culpa, mientras que el profesional tratante completó una ficha sociodemográfica con datos de la vulneración ocurrida. Los resultados sugieren que; abusos más intrusivos, crónicos, con empleo de fuerza e intrafamiliares se relacionan con mayores puntajes en los sentimientos de estigmatización, indefensión, traición y culpa. Adicionalmente, la edad de la víctima y el apoyo familiar ante la develación arrojaron correlaciones con el procesamiento traumatogénico de experiencias de violencia sexual.
\end{abstract}

Palabras clave: violencia sexual infantil, dinámicas traumatogénicas, victimología.

\begin{abstract}
The relation between the traumatogenic processing of sexual violence experienced by girls, with characteristics of suffered victimization is investigated. A sample of 40 girls, victims of sexual violence answered a scale of feelings of stigmatization, defenselessness, betrayal and guilt, while the treating professional completed a sociodemographic card with data extracted from the episodes of violation suffered. The results suggest that abuses performed with more intrusive, chronic, domestic and with use of force are related to highest scores in feelings of stigmatization, defenselessness, betrayal and guilt. In addition to this, the age of the victim and family support facing the disclosure generated correlations with the traumatogenic processing of sexual violence experiences.

Keywords: child sexual violence, traumatogenic dynamic, victimology.
\end{abstract}

\footnotetext{
Correspondencia dirigida a: Hugo Plaza Villaroel. Escuela de Psicología Universidad Santo Tomás, Av.1 Norte 3041, $7^{\circ}$ Piso, Viña del Mar. E-mail: hugoplaza@ santotomas.cl.
} 


\section{Introducción}

Desde una perspectiva cognitiva, se ha indagado en el rol de percepciones y atribuciones de las víctimas respecto del abuso sexual y su relación con síntomas y efectos de la violencia sexual. En un artículo clásico, Finkelhor y Browne (1985), señalan que existirían cuatro esquemas cognitivos, denominados "Dinámicas traumatogénicas"que mediarían entre el evento de abuso y el ajuste psicológico posterior. Estas dinámicas, alterarían la orientación cognitiva y emocional del niño hacia el mundo creando una distorsión en su capacidad afectiva, autoconcepto y visión de mundo.

Las dinámicas traumatogénicas descritas por Finkelhor et al. (1985) son: a) "sexualización traumática", refiere al proceso en que las víctimas evidencian un comportamiento y una preocupación sexual (interés o aversión) inapropiados para su edad, generando una conceptualización distorsionada acerca de las relaciones sexuales como resultado de cosas que los ofensores les ha dicho y hecho; b) "estigmatización", donde los niños y niñas que son víctimas a menudo se sienten aislados, culpables y avergonzados como resultado de este abuso; c) "Traición”, es una reacción sobre la pérdida de una figura significativa de confianza (en casos que el agresor sea conocido de la víctima), y que puede generar una intensa necesidad de lograr confianza y seguridad manifestando una extrema dependencia y búsqueda de una relación protectora o por el contrario, caracterizarse por la hostilidad, la ira, el aislamiento, agresividad y rechazo a las relaciones íntimas; y d) "Pérdida de control» (indefensión), que se relaciona con el miedo y ansiedad ante la creencia de incapacidad de controlar los acontecimientos nocivos, estas creencias ponen en peligro el sentido de eficacia y habilidades de afrontamiento. La sensación de pérdida de control podría asociarse a la desesperanza, depresión y conductas que podrían generar la incapacidad de hacer frente a su entorno, además de aumentar el riesgo de una victimización posterior.

En síntesis, es posible afirmar del modelo traumatogénico, que la estigmatización se asocia directamente con la culpa, la sexualización traumática se focaliza en los efectos en el comportamiento sexual, el sentimiento de traición se enfoca en las dificultades para formar relaciones $\mathrm{y}$, finalmente, la pérdida de control se relaciona con el miedo, ansiedad y sintomatologías asociadas al estrés post traumático.

En una misma línea teórica, Celano (1992), plantea un modelo de atribuciones de responsabilidad para niños y adolescentes víctimas, las cuales se resumen en un pensamiento global de auto desaprobación y culpa. Las atribuciones de culpa y responsabilidad se agrupan en las siguientes dimensiones: a) responsabilidad por no reconocer la interacción abusiva (ej. "no sabía que eso era abuso", "no sabía que esa forma de tocarme era inapropiada"; b) responsabilidad de participación (ej. "Nunca dije que se detuviera", "era como un juego"); c) responsabilidad por las consecuencias familiares de la develación (ej. "he causado problemas a mi familia", "ahora que conté lo ocurrido mi papá fue detenido"); d) responsabilidad por fallar en buscar ayuda (ej. "debí haber contado antes"); e) responsabilidad por haber obtenido placer o ganancias (ej. "yo recibía regalos por esto", "me decía que éramos como pololos"); y e) responsabilidad por no auto protegerse (ej. "Nunca supe que esto me iba a afectar después", "no supe defenderme"). Las atribuciones de responsabilidad serían un mediador entre el evento abusivo y las consecuencias psicológicas posteriores, no obstante, su génesis se relaciona tanto con la dinámica propia del abusador sobre la víctima, como en la respuesta del contexto familiar y/o institucional ante la develación de la víctima, adicionalmente incidiría el estilo atribucional de la víctima.

En tanto, Mannarino, Cohen y Berman (1994), plantean que niños y niñas víctimas, pueden desarrollar percepciones y atribuciones particulares sobre su experiencia de victimización. Estas atribuciones y percepciones incluyen: a) sentirse distinto a sus pares, en el sentido de creer que la condición traumática los diferencia del tránsito evolutivo de sus pares, en cuanto a un sentimiento de estigmatización que condiciona sus intereses, sus habilidades, su esquema corporal; b) autoinculpación por el abuso, relacionado con creencias de no haber podido evitar que el abuso ocurriera, por haber participado activamente de tales prácticas, de provocar consecuencias negativas para la familia a raíz de la develación del abuso; c) creencias de no ser veraces, asociado a la respuesta de la familia y el contexto de la víctima posterior a su develación, se trata de un sentimiento de baja credibilidad percibida; y d) reducción de la confianza interpersonal, frente a esta dimensión la víctima posterior al abuso establecería un patrón de evitación por establecer relaciones con otros, conductualmente se caracterizaría por evitación o agresión interpersonal.

Spaccarelli (1995), conceptualiza las creencias de la víctima asociadas al abuso sexual como un evento de transición o importante factor de estrés. En este sentido, la víctima no evalúa únicamente la interacción física concreta del abuso, sino que integra una 
serie compleja de eventos estresantes para configurar una experiencia ideográfica de la victimización. Este conjunto de eventos incluye no sólo los episodios de contactos abusivos, sino también comportamientos del agresor (ej., incentivos, manipulación, seducción, amenazas), las consecuencias ante la develación del abuso (ej. alteraciones en las relaciones familiares, la incredulidad familiar, la culpabilización de la víctima), y los eventos que se producen asociados a modos de victimización secundaria (ej. encuentros intrusivos con los servicios sociales, la policía, y particularmente, los profesionales del Derecho). La principal ventaja de este enfoque basado en eventos para evaluar el abuso sexual, es que se amplía el concepto de la gravedad de los abusos para incluir todas estas posibles fuentes de estrés, de modo que la evaluación primaria del evento traumático se encuentra amplificada por una serie de eventos asociados, explicando el potencial daño "traumatogénico"de la victimización sexual.

De modo más acotado, Feiring, Taska y Lewis (1996), se centran en el proceso de estigmatización. Entendiéndola como una dinámica que incluye evaluaciones de auto inculpación (principalmente una autoevaluación reprobatoria de ciertos comportamientos que permitieron que el abuso ocurriera) y baja autoestima, donde la vergüenza (que incluye sentimientos negativos sobre sí mismo, la imagen corporal, deseos de escapar o desaparecer) es la emoción básica para esta dinámica por sobre los sentimientos de culpa. Para Feiring et al. (1996), las explicaciones causales del abuso (atribución), juegan un rol central en el proceso de ajuste de la víctima, donde atribuciones internas, estables y globales serían un predictor de riesgo en el ajuste psicológico posterior al evento abusivo. De acuerdo a este modelo, el abuso sexual conduce a la vergüenza a través de la mediación de las atribuciones sobre el abuso, y a su vez la vergüenza conduce a un mal ajuste. Se plantea que el apoyo social, el género de la víctima y la etapa del desarrollo serían factores moderadores de los efectos de esta dinámica.

La evidencia empírica, ha señalado que estos mecanismos de creencias, percepciones y atribuciones negativas por parte de las víctimas sobre el evento traumático, su contexto próximo y sobre sí mismos, se asocian con diversa sintomatología clínica (Coffey, Leitenberg, Hening, Turner y Bennet, 1996; Cohen, Mannarino y Knudsen, 2005; Daigneault, Tourigni y Hebert, 2006; Tangney, Burggraf, y Wagner, 1995;
Steel, Sanna, Hammond, Whipple y Cross, 2004). Coffey, et al. (1996), realizando una continuidad al trabajo de Finkelhor et al. (1985), concluyeron que el nivel de malestar psicológico experimentado por las víctimas estaban mediatizados por los sentimientos de culpa y estigma percibido. Cantón-Cortés, Cantón, Justicia y Cortés (2011), sugieren que los factores cognitivos pueden ser más importantes que las características del abuso para predecir el ajuste psicológico de las víctimas, demostrando el rol mediador de las atribuciones de culpa (hacia sí mismo, otros y al propio agresor) en el bienestar psicológico.

Respecto de los factores que intervienen en la formación de los mecanismos cognitivos que procesan la experiencia de abuso, se ha planteado que las victimizaciones sexuales con mayor grado de intrusión y cronicidad se relacionan a un mayor empleo de percepciones y atribuciones de la violencia sufrida (Feiring y Cleland, 2007; Quas, Goodman y Jones., 2003). Adicionalmente se ha planteado que a mayor edad de la víctima es un predictor de un mayor empleo de estas dinámicas (Celano, 1992; Spaccarelli, 1995; Feiring et al., 1996). Con menor evidencia, se ha postulado el rol del apoyo familiar frente a la develación (Celano, 1992; Mannarino et al, 1994), la familiaridad del agresor con la víctima (McLean y Gallop, 2003) y la dinámica abusiva por parte del agresor (Feiring et al, 2007; Spaccarelli 1995) en la organización de estos mecanismos cognitivos.

El propósito del presente estudio es examinar la relación entre los factores asociados a la victimización (variables victimológicas) con el empleo de dinámicas cognitivas traumatogénicas por parte de víctimas de violencia sexual infantil. Para lo anterior se consideran las siguientes hipótesis: H1) victimizaciones más intrusivas (abuso, violación) se asocian a un mayor puntaje en las dinámicas cognitivas; H2) existe una relación directa entre la frecuencia de la victimización sexual y las dinámicas cognitivas; H3) a mayor grado de familiaridad entre agresor y víctima existe un incremento en las puntuaciones de las dinámicas cognitivas; H4) las estrategias de acoso de parte del agresor (engaño, uso de fuerza) se relacionan de modo diferencial con las dinámicas cognitivas; H5) mayor edad de la víctima correlaciona de modo directo con las puntuaciones en las dinámicas cognitivas; y H6) mayor apoyo familiar frente a la develación se relaciona con menores puntuaciones en las dinámicas cognitivas. 


\section{Método}

\section{Participantes}

Se emplea un muestreo intencionado compuesto por 40 niñas víctimas de violencia sexual, pertenecientes a centros de atención y reparación en maltrato infantil grave de la Red del Servicio Nacional de Menores (en adelante red SENAME), de las ciudades de Viña del Mar y Valparaíso, Chile. De entre 9 y 17 años (20\% entre 9 y 11 años, 52,5\% entre 12 y 14 años y $27,5 \%$ entre 15 y 17 años). Del total de participantes en el estudio, un $56,4 \%$ ha sido víctima de abuso sexual y un $43,6 \%$ víctima del delito de violación, en todos los casos los agresores fueron varones. Los casos seleccionados cumplieron con los siguientes criterios de inclusión: a) niñas con edades desde los ocho a los dieciocho años; b) experiencia de victimización respaldada por informes médicos y/o psicológicos; c) sus causas se encontraran judicializadas en tribunales de familia o bien en el Ministerio Público; d) que en cada caso, las niñas ya hayan reportado relato al terapeuta respecto de la vulneración ocurrida; e) que contaran con acompañamiento terapéutico; f) que previamente los y las profesionales tratantes evaluaran la conveniencia de la aplicación de los instrumentos, en términos de no interferir en el proceso terapéutico; g) que sus padres o tutores responsables firmaran el respectivo consentimiento informado; h) que las mismas niñas aceptaran participar en la investigación. Fueron criterios de exclusión: a) presentar analfabetismo o algún grado de retardo mental; b) la ausencia de relato de la vulneración ocurrida; c) que el profesional tratante determinara la inconveniencia de la administración de los instrumentos; d) la negativa de los padres al consentimiento informado, e) la negativa de las niñas de participar en la investigación.

\section{Instrumentos}

Cuestionario Sociodemográfico sobre Violencia Sexual Infantil. Este cuestionario, desarrollado por los autores, para efectos específicos de la presente investigación, recogió información sociodemográfica y características de la violencia sexual sufrida (variables victimológicas) de forma anónima, y fue respondida por los terapeutas a cargo de cada una de las participantes. Respecto de las características de la violencia sexual sufrida, se consideraron las siguientes variables: a) relación con el agresor (intrafamiliar, extra familiar); b) tipo de victimización (abuso sexual, violación); c) frecuencia de la victimización sexual (episodio único, ocasional, victimización crónica); d) apoyo familiar ante la develación del abuso (no dar credibilidad y culpar a la víctima, creer en la develación pero mantener contacto con agresor, creer y proteger activamente a la víctima); y e) estrategias de acoso de parte del agresor (estrategias de engaño y/o seducción, estrategias punitivas con uso de fuerza física y/o verbal).

Escala de sentimientos de Estigmatización, Traición, Culpa e Indefensión (Coffey et al., 1996). Se trata de una escala de 18 ítems en formato de respuesta Likert, creada para valorar cuatro dinámicas cognitivas asociadas a la victimización sexual. Su versión original en inglés consta de las siguientes dimensiones con las siguientes propiedades psicométricas: Estigmatización (4 ítems, con una consistencia interna de 0.78), Traición (4 ítems, con una consistencia interna de 0.91), Indefensión (3 ítems, con una consistencia interna de 0.94) y Culpa (7 ítems, con una consistencia interna de 0.82 ). Dado que para el presente estudio no se contaba con una medida de cogniciones adecuada para estos efectos, se procedió a realizar una adaptación preliminar de esta escala al idioma castellano. Para tal efecto se empleó el enfoque de comité (Brislin, 1980) con descentramiento (Werner y Campbell, 1970) traduciendo la escala del inglés al castellano, de modo independiente, por tres profesionales con las cuales se elaboró una versión única del instrumento, esta versión fue a su vez re-traducida al idioma inglés para contrastar su equivalencia semántica. Se asignaron cinco opciones de respuesta a cada uno de los ítems desde "nunca"a "siempre", y el puntaje de cada una de las subescalas se obtuvo sumando las respuestas de cada uno de los ítems y dividiendo el resultado según el número de reactivos de cada dimensión (en la tabla 1 se muestra un ejemplo de los ítems por cada dimensión).

Una vez aplicada la escala a la muestra, se examinó su consistencia interna, arrojando los siguientes resultados: Estigmatización (alfa 0.55), Traición (alfa 0.75), Indefensión (alfa 0.82) у Culpa (alfa 0.83).

\section{Procedimiento}

A modo de resguardar la integridad de las participantes y del posible malestar o daño que podía surgir como efecto de la administración de la Escala de sentimientos de Estigmatización, Traición, Culpa e Indefensión, se emplearon los siguientes criterios de resguardo: a) el equipo de investigación presentaba competencia en la temática a investigar, puesto que en el contexto de su ejercicio profesional mantienen 
Tabla 1.

Ejemplo de ítem por cada dimensión de la escala.

Tabla 2.

Descriptivos variables victimológicas $(n=40)$.

\begin{tabular}{ll}
\hline Variable & Porcentaje \\
\hline Relación con agresor. & $38.5 \%$ \\
Vive con víctima. & $61.5 \%$ \\
No Vive con víctima. & \\
Tipo Victimización. & \\
Abuso Sexual. & $54.6 \%$ \\
Violación. & $43.6 \%$ \\
Frecuencia Victimización. & \\
Episodio único. & $27.5 \%$ \\
Victimización Ocasional. & $30 \%$ \\
Victimización Crónica. & $42.5 \%$ \\
Apoyo familiar ante develación de la víctima. & \\
No creen y culpan a la víctima. & $17.6 \%$ \\
Creen, pero mantienen contacto con agresor. & $23.5 \%$ \\
Creen y protegen a la víctima & $58.8 \%$ \\
Estrategias de acoso empleadas por agresor. & \\
Estrategias de engaño. & $43.4 \%$ \\
Estrategias punitivas. & $56.6 \%$ \\
\hline
\end{tabular}

contacto con población infanto-juvenil victimizada; b) como criterio esencial de reclutamiento de las participantes, se consideró la opinión del profesional tratante respecto de riesgo y beneficio potencial que representaba para cada una de las niñas el hecho de participar en la investigación; c) la aplicación se realizó en un espacio seguro, esto es, en el mismo setting terapéutico donde las niñas asisten a terapia reparatoria, adicionalmente, la administración por parte de los mismos terapeutas evitó el contacto de las niñas con el equipo investigador; d) se solicitó consentimiento y asentimiento a los padres y/o tutores legales, y a las mismas niñas; e) se informó a las niñas de su derecho de dejar de responder al instrumento de evaluación en caso que les provocara incomodidad o malestar; f) cada terapeuta podía suspender la aplicación del instrumento de evaluación en caso que advirtiera incomodidad o malestar en las niñas; g) la información obtenida por medio del instrumento de evaluación era devuelta al terapeuta con la finalidad de aportar en su proceso de trabajo reparatorio; y h) los datos sociodemográficos, de victimización y las respuestas a los ítems del instrumento de evaluación fue computada en un conjunto de datos, de modo anónimo, sin hacer referencia alguna respecto de datos de identificación directa.

Con los criterios anteriormente expuestos, se solicitó autorización para la realización de la investigación a los centros de atención y reparación en maltrato infantil grave de la red SENAME, de las ciudades de Viña del Mar y Valparaíso. En esta etapa, se explicaron los procedimientos, las características de la muestra y se exhibió la batería de pruebas a aplicar.

Una vez conseguidas las autorizaciones, a cada psicólogo o psicóloga le fueron entregados: los criterios 
de inclusión, el set de baterías y una explicación del procedimiento de administración, donde cada terapeuta seleccionó casos que cumplieran con los criterios de inclusión y que pudieran responder a los instrumentos.

Por su parte, a cada padre, madre o adulto responsable de los casos seleccionados, se le solicitó su consentimiento informado para que su hija o pupila pudiera participar de la investigación, resguardando su derecho a confidencialidad y disentimiento de participar de la aplicación. En términos generales el consentimiento informado señaló que el psicólogo o psicóloga responsable de la atención de su hija o representada contestaría una ficha demográfica (compuesta por datos como; edad, género y antecedentes generales del tipo de vulneración sufrida) y que la niña respondería una escala sobre pensamientos y sentimientos respecto de la vulneración sufrida acompañado por su psicólogo/a tratante (en cada consentimiento se adjuntó el instrumento de evaluación a aplicar).

Una vez obtenido el consentimiento informado, cada psicólogo o psicóloga, consultó a cada niña respecto de su acuerdo en responder la escala, bajo la consigna general que respondería un cuestionario sobre pensamientos y sentimientos respecto de la victimización sufrida, que estos datos serían incluidos en una investigación sin identificar su nombre, que tenía derecho a no responder, y que en caso que aceptara y durante la administración se sentía incómoda podía dejar de responder. Posterior a esto, se procedió a la administración y al llenado de la ficha sociodemográfica de victimización infantil. Los datos obtenidos fueron tabulados en una base de datos y luego analizados en el software estadístico SPSS 18.0, mientras que los cálculos de tamaños de efecto fueron realizados con el software Gpower 3.1.

Tabla 3.

Descriptivos escala de Estigmatización, Traición, Impotencia y Culpa $(n=40)$.

\begin{tabular}{lll}
\hline & Media & DT \\
\hline Estigmatización & 2,42 & 0,87 \\
Traición & 2,5 & 1,15 \\
Impotencia & 2,79 & 1,33 \\
Culpa & 2,07 & 0,98 \\
\hline
\end{tabular}

\section{Tabla 4.}

Promedios $(M)$ y desviaciones típicas $(D T)$, de puntajes de dinámicas cognitivas según tipo legal de violencia sufrida y t de Student para muestras independientes $(n=40)$.

\begin{tabular}{lcccc}
\hline Dinámica Cognitiva & Abuso Sexual $(\mathrm{n}=22)$. & $\begin{array}{c}\text { Violación } \\
(\mathrm{n}=18)\end{array}$ & $t(38)$ & d de Cohen. \\
\hline Estigmatización & $2,09(0,70)$ & $2,83(0,92)$ & $-2,846 * *$ & 0,9 \\
Traición & $2,33(1,21)$ & $2,687(1,1)$ & $-0,923$ & 0,3 \\
Impotencia & $2,52(1,28)$ & $3,08(1,36)$ & $-1,326$ & 0,4 \\
Culpa & $1,85(0,86)$ & $2,37(1,10)$ & $-1,654 *$ & 0,5 \\
\hline
\end{tabular}

$* p<0,05 . * * p<0,01$. Prueba de significación unilateral.

Tabla 5.

Promedios de puntajes $(M)$ y desviaciones típicas $(D T)$ de las dinámicas cognitivas, según frecuencia en la exposición a violencia sexual y ANOVAs de linealidad $(n=40)$.

\begin{tabular}{lcclcc} 
Dinámica Cognitiva & $\begin{array}{c}\text { Episodio único } \\
(\mathrm{n}=11)\end{array}$ & Ocasional $(\mathrm{n}=12)$ & Crónico $(\mathrm{n}=17)$ & $F(2)$ & Eta cuadrado. \\
\hline Estigmatización & $1,96(0,46)$ & $2,39(0,89)$ & $2,76(0,94)$ & $3,45 *$ & 0,157 \\
Traición & $2,15(0,95)$ & $2,23(1,29)$ & $2,93(1,10)$ & 2,16 & 0,104 \\
Impotencia & $2,53(1,29)$ & $2,64(1,52)$ & $3,08(1,24)$ & 0,699 & 0,036 \\
Culpa & $1,76(0,54)$ & $1,95(1,03)$ & $2,36(1,15)$ & 1,454 & 0,073 \\
\hline
\end{tabular}

$* p<0,05$. Prueba de significación unilateral. 


\section{Resultados}

El análisis de frecuencia de las variables asociadas a la victimización (Tabla 2), muestra que; un 38,5\% de los agresores vivía con la víctima, mientras que un $61.5 \%$ eran conocidos de la víctima pero no vivían con ella, no contabilizándose casos de agresores desconocidos. Según el tipo legal de victimización, el 54.6\% de la muestra sufrió de abuso sexual, mientras que un $43.6 \%$ fue víctima del delito de violación. En cuanto a la frecuencia de la victimización sexual, un $42.5 \%$ sufrió victimización crónica, un $30 \%$ ocasional, y un
$27.5 \%$ en episodio único. Las estrategias de acoso empleadas por parte del agresor fueron mayoritariamente de orden punitivo (violencia física o verbal) con un $56.6 \%$, mientras que un $43.4 \%$ sufrió de acoso basado en engaño y/o seducción. Finalmente, en apoyo familiar a la víctima ante la develación, el $58 \%$ recibió apoyo consistente en otorgar credibilidad a su relato $\mathrm{y}$ adicionalmente mantener distancia del agresor, un $23.5 \%$ recibe apoyo ambivalente, es decir, por una parte la familia cree lo reportado pero mantienen contacto con agresor, mientras que un $17.6 \%$ no recibe apoyo adecuado, que implica que la familia no cree en la

Tabla 6.

Promedios (M) y desviaciones típicas (DT), de puntajes de Dinámicas cognitivas según familiaridad agresor - víctima y t de Student para muestras independientes $(n=40)$.

\begin{tabular}{lllll}
\hline Dinámica Cognitiva & $\begin{array}{l}\text { Vive con víctima } \\
(\mathrm{n}=16) .\end{array}$ & $\begin{array}{l}\text { No vive con víctima. } \\
(\mathrm{n}=24)\end{array}$ & $t(38)$ & $\mathrm{d}$ de Cohen. \\
Estigmatización & $2,45(0,89)$ & $2,39(0,87)$ & 0,210 & 0,07 \\
Traición & $2,87(1,22)$ & $2,24(1,08)$ & $1,68 *$ & 0,55 \\
Impotencia & $2,91(1,24)$ & $2,67(1,4)$ & 0,55 & 0,18 \\
Culpa & $2,27(1,19)$ & $1,96(0,85)$ & 0,94 & 0,29 \\
\hline
\end{tabular}

${ }^{*} \mathrm{p}<0,05$. Prueba de significación unilateral.

Tabla 7.

Promedios $(M)$ y desviaciones típicas (DT), de puntajes de dinámicas cognitivas, según tipo de estrategias del agresor y t de Student para muestras independientes $(n=40)$.

\begin{tabular}{|c|c|c|c|c|}
\hline Dinámica Cognitiva & $\begin{array}{l}\text { Estrategias de engaño } \\
\text { y seducción }(\mathrm{n}=18)\end{array}$ & $\begin{array}{l}\text { Estrategias punitivas } \\
(\mathrm{n}=22)\end{array}$ & $t(38)$ & $\mathrm{d}$ de Cohen. \\
\hline Estigmatización & $2,29(0,84)$ & $2,46(0,91)$ & $-0,411$ & 0,19 \\
\hline Traición & $2,54(1,43)$ & $2,57(1,11)$ & $-0,56$ & 0,02 \\
\hline Impotencia & $1,89(0,98)$ & $2,95(1,33)$ & $-1,855 *$ & 0,9 \\
\hline Culpa & $2,26(0,89)$ & $2,07(1,02)$ & 0,425 & 0,19 \\
\hline
\end{tabular}

${ }^{*} p<0,05$. Prueba de significación unilateral.

Tabla 8.

Correlaciones simples (rho de Spearman), entre puntajes Dinámicas Cognitivas con edad de la víctima y apoyo familiar ante la develación. $(n=40)$.

\begin{tabular}{lll}
\hline & Edad Víctima & Apoyo Develación \\
Estigmatización & $0.363^{*}$ & $-0.367 *$ \\
Traición & 0.120 & -0.158 \\
Impotencia & 0.172 & $-0.464 * *$ \\
Culpa & $0.425 * *$ & -0.096 \\
\hline
\end{tabular}

${ }^{*} \mathrm{p}<0,05 .{ }^{* *} \mathrm{p}<0,01$. Prueba de significación unilateral. 
develación de la niña y adicionalmente mantiene el contacto con el agresor.

Los análisis descriptivos de la escala de dinámicas cognitivas (Tabla 3), muestran las siguientes puntuaciones en orden descendente: Impotencia (media de 2.79 y desviación típica de 1,33), Traición (media de 2,5 y desviación típica de 1,15), Estigmatización (media de 2,42 y desviación típica de 0,87), y Culpa (media de 2.07 y desviación típica de 0.98 ).

Respecto de la relación entre el nivel de intrusión de la victimización sexual (abuso sexual o violación) y el empleo de las dinámicas cognitivas (H1), se procedió a examinar si existían diferencias entre el empleo de las dinámicas y el tipo de victimización (abuso sexual o violación), para lo anterior, se contrastó con una prueba t para muestras independientes. De acuerdo a la tabla 4, en primer lugar es posible observar que todas las dinámicas cognitivas tienen puntuaciones más altas para los casos de violación en comparación con los de abuso sexual, las diferencias estadísticamente significativas son para las dinámicas de estigmatización ( $\mathrm{t}(38)=-2,846, p<$ $0,01)$, con un tamaño de efecto "grande"y culpa $(\mathrm{t}(38)$ $=-1,654, p<0,05)$, con un tamaño de efecto "mediano".

Examinando la relación entre la frecuencia de la victimización y el empleo de las dinámicas cognitivas $(\mathrm{H} 2)$, se observa en la tabla 5, que mientras mayor tiende a ser la frecuencia de exposición de la víctima a violencia sexual, mayor tienden a ser las puntuaciones de las dinámicas cognitivas. La dinámica de estigmatización arroja una relación lineal significativa $(\mathrm{F}(2)$ $=3,45, p<0,05)$ con respecto de la frecuencia de la victimización, con un tamaño de efecto "grande".

Para contrastar las puntuaciones de las dinámicas cognitivas con el grado de familiaridad entre agresor y víctima (H3), se empleó la prueba $t$ de Student para muestras independientes. Los resultados de la tabla 5 , arrojan puntuaciones más altas en las dinámicas cognitivas para aquellos casos en los que existe un contacto cotidiano entre la víctima y agresor (vive con la víctima). Se observó una puntuación significativamente mayor en la dinámica de traición $(\mathrm{t}(38)=1,68$, $p<0,05)$, con un tamaño de efecto "mediano", en las víctimas que vivían con el agresor en comparación con las que no mantenían este contacto cotidiano.

$\mathrm{Al}$ analizar la relación entre el tipo de estrategias de acoso de parte del agresor y las puntuaciones de las dinámicas cognitivas, la prueba $t$ de Student para muestras independientes arrojó diferencias significativas. En efecto, la tabla 7 señala que la dinámica de impotencia es significativamente más alta $(\mathrm{t}(38)=-1,855, p<$ $0,05)$ cuando el agresor ha implementado estrategias punitivas sobre la víctima (como violencia física y amenazas); adicionalmente el tamaño del efecto para esta diferencia se considera "grande". Continuando con este análisis, resulta relevante señalar que la dinámica de culpa exhibe una puntuación mayor con el uso de estrategias de engaño y seducción por parte del agresor, aun cuando no arroja diferencias significativas, esta tendencia parece orientada hacia lo teóricamente expuesto.

Finalmente para examinar la relación entre las puntuaciones de las dinámicas cognitivas con la edad de la víctima (H5) y el grado de apoyo familiar ante la develación del abuso (H6), se empleó correlación de Spearman (dado que estas dos últimas variables fueron medidas en un nivel ordinal). Tal como se aprecia en la tabla 8, la edad de la víctima y el apoyo familiar ante la develación correlacionan de modo esperado. La edad de la víctima, se asocia directamente con estigmatización (r. 0.36) y culpa (r. 0.42). Por su parte, el apoyo familiar ante la develación exhibe una relación inversa con estigmatización (r. -0.36) e impotencia (r.-0.46).

\section{Discusión}

La presente investigación se propuso examinar seis hipótesis respecto de la relación entre dinámicas cognitivas propias de la victimización sexual con variables victimológicas y sociodemográficas. Para ello, se tradujo y adaptó una escala ad hoc para este propósito; la versión chilena de esta escala fue aplicada, junto a una ficha demográfica, a una muestra femenina de víctimas de violencia sexual infantil, de las ciudades de Valparaíso y Viña del Mar.

Los resultados obtenidos permiten, aunque de modo no generalizado, apoyar las hipótesis planteadas en la investigación. En primer término, las cogniciones referidas a estigmatización demuestran resultados compatibles con la evidencia empírica, dado que, estarían asociadas a victimizaciones más intrusivas y de mayor cronicidad (Feiring et al., 2007; Quas et al., 2003), en víctimas de mayor edad (Celano, 1992; Spaccarelli, 1995; Feiring et al., 1996) y ante un menor apoyo familiar ante la develación (Celano, 1992; Mannarino et al, 1994). Lo anterior resulta relevante comprenderlo en el contexto de esta dinámica particular dado que victimizaciones más intrusivas, crónicas e incompatibles con el tránsito evolutivo de la víctima, pueden asociarse a sentimientos de estas niñas de ser "distintas"respecto de sus pares, a un deterioro en su imagen corporal, donde la vergüenza como emoción básica inhibe su ajuste posterior a la victimización (ej. negación de la experiencia, evitar 
hablar de la experiencia, activación emocional al oír conversaciones cuyo contenido sea la sexualidad, evitar la develación). La edad como factor asociado guardaría relación con las capacidades verbales y cognitivas para elaborar la experiencia en base a esquemas de mayor complejidad que, en este caso, podrían estar a la base respecto que las percepciones de estigmatización tengan cualidades más robustas e identificables en el pensamiento de estas niñas, además por la naturaleza de esa etapa del desarrollo, tienen mayor labilidad emocional (Dryfoos, 1990; Smetana, 1989; Smetana, Yau y Hanson, 1991) y, en su tarea vital de búsqueda de identidad, el componente de estigmatización podría integrarse de modo negativo en su autoconcepto. El apoyo social ante la develación (entendido en las dimensiones credibilidad ante la develación y distancia protectora respecto del agresor), también arrojó relaciones significativas con la dinámica de estigmatización, reflejando que la percepción de credibilidad y protección en las víctimas sería un factor relevante en la elaboración de esta dinámica. Esto, pues, en la medida en que las niñas perciben que su relato es creíble y además, como resultado concreto, su ambiente familiar protege distanciando al agresor, habría un menor grado en los sentimientos de estigmatización.

En segundo lugar la dinámica de traición se vinculó a victimizaciones ocurridas en el contexto intrafamiliar, coincidiendo este resultado con investigaciones anteriores (McLean et al., 2003). Al respecto, es posible formular que cuando la victimización ocurre en el contexto familiar se asocia con sentimientos de rechazo o ansiedad por establecer relaciones con otros, ante la pérdida de confianza por sus figuras significativas, alterando el espacio de seguridad vincular básica de las niñas, relacionado al hecho que el agresor era supuestamente un agente que debía ofrecer protección y seguridad (Finkelhor et al., 1985; Mannarino et al., 1994).

En tercer lugar, el sentimiento de impotencia se asocia con un bajo apoyo familiar ante la develación (Celano, 1992; Mannarino et al, 1994) y con estrategias punitivas (Feiring et al, 2007; Spaccarelli 1995) de parte del agresor. Conforme a lo anterior, victimizaciones ocurridas en el contexto de dinámicas abusivas de orden punitivo, contribuirían a un procesamiento atribucional de incontrolabilidad de eventos potencialmente dañinos (indefensión), disminuyendo de este modo el sentimiento de autoeficacia (desamparo) y el despliegue de estrategias de afrontamiento más activas (Cantón et al., 2003). Adicionalmente, una respuesta inadecuada del contexto familiar ante la develación (como no creer y mantener el contacto con el agresor), puede resultar en un proceso de invalidación de las estrategias de ajuste de la víctima, incrementando el sentimiento de impotencia. Como ya fue señalado, esta dinámica se vincula con emociones como miedo, ansiedad (hipervigilancia) y síntomas depresivos.

En cuarto lugar, las cogniciones referidas a la culpa son mayores en relación con una mayor edad de la víctima, y con victimizaciones más intrusivas (violación). Las atribuciones de responsabilidad por la victimización, incluyen tanto pensamientos de ineficacia por hacer frente al abuso como pensamientos de participación voluntaria en la dinámica abusiva (Celano, 1992; Feiring et al., 1996). De este modo, victimizaciones intrusivas como la violación bien pueden activar pensamientos de ineficacia en: la autoprotección, el reconocimiento de los patrones abusivos, las consecuencias de develación del abuso y la frecuencia de las agresiones, donde la edad puede ser un factor adicional que contribuya a una elaboración más compleja de los esquemas auto- reprobatorios en el pensamiento de las niñas. Se ha postulado que la autoinculpación se relacionaría con el estrés postraumático al activar una evaluación reiterada del evento, seleccionando aquellos comportamientos que justifiquen este sentimiento culposo (recuerdos invasivos) (Daigneault et al., 2006; Steel et al., 2004). Finalmente, aun cuando no se obtuvieron relaciones significativas, se observó un puntaje más alto en la dinámica de culpa frente a estrategias de engaño de parte del agresor (como la seducción). Se postula que ante tal dinámica, la víctima podría asumir una valoración más amplia de responsabilidad por lo ocurrido (Spaccarelli, 1995), en el sentido de percibir que ha participado de modo activo.

Es importante considerar para este estudio al menos las siguientes limitaciones. En primer lugar, si bien se ha empleado un instrumento adecuado para evaluar cogniciones asociadas a la victimización sexual, sus propiedades psicométricas no poseen amplia evidencia en nuestro país, pese a que sus escalas arrojaron adecuados índices de confiabilidad y se infieren evidencias de validez debido a la correlación esperada de sus puntuaciones con variables criterio. Adicionalmente, es relevante señalar que el instrumento empleado, alude directamente a la victimización sufrida, lo cual exige acompañamiento terapéutico en su administración por la posibilidad de victimización secundaria. Esto se debe tener en cuenta tanto en la decisión de exponer al niño o niña al material de la escala, en la administración por parte del terapeuta, en la supervisión de reacciones de incomodidad por parte del niño o niña, y finalmente en contextualizar la aplicación dentro de un proceso 
reparatorio respecto de los sentimientos y pensamientos asociados a estas vulneraciones.

Otra limitación refiere, al tamaño muestral (justificado en la dificultad natural de acceder a casos sexualmente vulnerados), el que no permite la generalización de los resultados, no obstante, se obtuvo una potencia estadística adecuada en todas las inferencias con tamaño de efecto grande. Debido al número de casos, no se contrastó la variable género (dado que la cantidad de niños era insuficiente para el análisis), por lo cual el efecto género en las dinámicas cognitivas, debiese ser abordado en investigaciones venideras.

La presente investigación constituye una aproximación empírica frente a percepciones y atribuciones asociadas a la victimización sexual infantil en Chile. Por esta razón se hace necesario continuar estudiando el tema, evaluando los hallazgos aquí logrados, identificando nuevas relaciones y/o subsanando los aspectos metodológicos ya señalados. Lo anterior podría proporcionar beneficios, al menos, en dos direcciones: a) examinando en profundidad las propiedades psicométricas de la escala de Estigmatización, Traición, Culpa e Indefensión, y/o b) desarrollando investigaciones que comprueben el efecto mediador que tienen las atribuciones y percepciones que hace la víctima sobre el evento traumático y su posterior ajuste psicológico, incrementando así el cuerpo de conocimiento en nuestro país sobre este fenómeno traumático.

\section{Referencias}

Brislin, R. W. (1980). Translation and content analysis of oral and written material. En H. C. Triandis \& J. W. Berry (Eds.), Handbook of cross-cultural psychology (pp. 389-444). Boston: Allyn and Bacon.

Cantón-Cortés, D., Cantón, J., Justicia, F. \& Cortés, M. (2011). Un modelo de los efectos del abuso sexual infantil sobre el estrés post-traumático: el rol mediador de las atribuciones de culpa y afrontamiento de evitación. Psicothema, 23(1), 66-73. Recuperado de http://www.psicothema.com/pdf/3851.pdf

Celano, M. (1992). A developmental model of victim's internal attributions of responsibility for sexual abuse. Journal of interpersonal violence, 7(1), 57-69. doi:10.1177/088626092007001005

Coffey, P., Leitenberg, H., Hening, K., Turner, T., \& Bennet, R. (1996). Mediators of the long-term impact of child sexual abuse: perceived stigma, betrayal, powerlessness, and self-blame. Child Abuse and Neglect, 20(5), 447-455. doi:10.1016/01452134(96)00019-1

Cohen, J., Mannarino, A. \& Knudsen, K. (2005).Treating sexually abused children: 1 year follow-up of a randomized controlled trial. Child Abuse Neglect, 29(2), 135-45. doi:10.1016/j.chiabu.2004.12.005

Daigneault, I., Tourigni, M. \& Hebert, M. (2006). Self-Attributions of Blame in Sexually Abused Adolescents: A Mediational Model. Journal of Traumatic Stress, 19(1), 153-157. doi:10.1002/jts.20101
Dryfoos, J. (1990). Adolescent at risk: Prevalence and prevention. New York: Oxford University Press.

Feiring, C. \& Cleland, C. (2007). Childhood sexual abuse and abuse specific attributions of blame over 6 years following discovery. Child Abuse and Neglect, 31(11-12), 1169-1186. doi:10.1016/j. chiabu.2007.03.020

Feiring, C., Taska, L. \& Lewis, M. (1996). A process model for understanding adaptation to sexual abuse; the role of shame in defining stigmatization. Child Abuse and Neglect, 20(8), 767-782. doi:10.1016/0145-2134(96)00064-6

Finkelhor, D. \& Browne, A. (1985). The traumatic impact of child abuse: A conceptualization. American Journal of Orthopsychiatry, 55(4), 530-541. doi:10.1111/j.1939-0025.1985.tb02703.x

McLean, L. \& Gallop, R. (2003). Implications of childhood sexual abuse for adult borderline personality disorder and complex posttraumatic stress disorder. American Journal of Psychiatry, 160(2), 369-371. doi:10.1176/appi.ajp.160.2.369

Mannarino, A., Cohen J. \& Berman S. (1994). The Children's Attributions and Perceptions Scale, a new measure of sexual abuse related factors. Journal of Consulting and Clinical Psychology, 23(2), 204-211. doi:10.1207/s15374424jccp2302_9

Quas, J., Goodman, S. \& Jones, D. (2003). Predictors of attributions of self-blame and internalizing behavior problems in sexually abused children. Journal of Child Psychology and Psychiatry, 44(5), 723-736. doi:10.1111/1469-7610.00158

Smetana, J. (1989). Adolescents' and parents' reasoning about actual family conflict. Child Development, 60(5), 1052-1067. doi:10.2307/1130779

Smetana, J., Yau, J. \& Hanson, S. (1991). Conflict resolution in families with adolescents. Journal of Research on Adolescence, 1(2), 189-206. doi:10.1207/s15327795jra0102_5

Spaccarelli, S. (1995). Measuring abuse stress and negative cognitive appraisals in child sexual abuse: Validity data on two new scales. Journal of Abnormal Psychology, 23(6), 703-72. doi:10.1007/ BF01447473

Steel, J., Sanna, L., Hammond, B., Whipple, J. \& Cross, H. (2004). Psychological sequelae of childhood sexual abuse: Abuse-related characteristics, coping strategies and attributional style. Child Abuse and Neglect, 28(7), 785-801. doi:10.1016/j.chiabu.2003.12.004

Tangney, J., Burggraf, S. \& Wagner, P. (1995). Shame-proneness, guilt-proneness and psychological symptoms. En J. P. Tangney $\&$ K. W. Fischer (Eds.), Self-conscious emotions: The psychology of shame, guilt, embarrassment and pride (pp. 343-367). New York: Guilford Press.

Werner, O. \& Campbell, D. (1970). Translating working through interpreters, and the problem of decentering. En R. Narroll \& R. Cohen (Eds.), A handbook of method in cultural anthropology (pp. 398-420). Nueva York: American Museum of Natural History Press. 